\title{
Generation and Application of a Probabilistic Breast Cancer Atlas
}

\author{
Daniel B. Russakoff and Akira Hasegawa \\ Fujifilm Software (California), Inc., San Jose, California \\ drussakoff@fujifilmsoft.com
}

\begin{abstract}
Computer-aided detection (CAD) has become increasingly common in recent years as a tool in catching breast cancer in its early, more treatable stages. More and more breast centers are using CAD as studies continue to demonstrate its effectiveness. As the technology behind CAD improves, so do its results and its impact on society. In trying to improve the sensitivity and specificity of CAD algorithms, a good deal of work has been done on feature extraction, the generation of mathematical representations of mammographic features which can help distinguish true cancerous lesions from false ones. One feature that is not currently seen in the literature that physicians rely on in making their decisions is location within the breast. This is a difficult feature to calculate as it requires a good deal of prior knowledge as well as some way of accounting for the tremendous variability present in breast shapes. In this paper, we present a method for the generation and implementation of a probabilistic breast cancer atlas. We then validate this method on data from the Digital Database for Screening Mammography (DDSM).
\end{abstract}

\section{Introduction}

Computer-aided detection (CAD) of breast cancer in mammography has been shown to be a very effective tool in the fight against breast cancer. One recent study of a regional mammography screening program with and without CAD showed an increase in the cancer detection rate, a decrease in the mean age at screening detection, and a much earlier detection of invasive cancers using CAD [1. These results corroborate the findings of a number of other, earlier studies that indicate the usefulness of CAD 233 .

The basic algorithms for CAD systems in mammography are well documented [4] and well understood. The pipeline consists of three steps:

1. Detection of potential lesion candidates.

2. Feature extraction from this pool of candidates.

3. Classification of these candidates based on the extracted features.

While steps 1 and 3 are important to be sure, step 2 is the most vital as it, more than the others, is where the algorithm mimics the experience of the radiologist. In general, the features extracted in step 2 are all characteristics of cancerous lesions that radiologists themselves have described as being useful for detection 
of cancer [5]. Among the features typically calculated in feature extraction are those local to a neighborhood around the candidate lesion including: degree of spiculation, shape of the border, strength of the margin, pixel density, contrast with background, and texture 4. Also calculated are global features such as: left-right symmetry, temporal stability, and multiple view correlation [4]. None of these features, however, takes into account one of the most important cues a radiologist uses, namely location information and its influence on cancer probabilities. There is a great deal of well-documented data indicating that certain areas of the breast are much more likely to have cancer [6] than others. One oft-cited study is due to Hanley [8] and suggests that over $50 \%$ of cancer can be found in the upper half of the breast. CAD algorithms hoping to mimic experienced radiologists must take this information into account. To do so, we propose to generate a set of probabilistic breast cancer atlases.

There exist, however, many difficulties that must be overcome before we can effectively generate this information and add it into an algorithm. The rest of this paper details a method for handling these issues on the way towards a functioning probabilistic atlas. Section 2 will detail our use of shape modelling to generate a common reference space in which to create our atlases. Section 3 will further describe our shape parameterization together with our atlas generation procedure. And, finally, Section 4 will detail how we use the atlas in a CAD algorithm and present our results on the Digital Database for Screening Mammography (DDSM) 9 .

\section{Shape Analysis}

One of biggest challenges in defining a breast cancer atlas is the large amount of variability associated with the shapes and sizes of breasts. To overcome this, we must find a way to equate, normalize, and account for this variation. Here we make our first simplifying assumption; namely that we are only interested in accounting for the variations in the silhouettes of the breasts as opposed to their appearances. Here we also assume that we have a reliable algorithm to segment the breast area from the background in a standard mammogram. Though a nontrivial problem in and of itself, there are a number of effective algorithms in the literature that can be used for this purpose (e.g. [10]). Since a breast silhouette is a closed contour, we can parameterize its shape as a chain code together with a center of mass as shown in Figure $3 \mathrm{~b}$. Now, in order to generate an atlas in some common reference frame, what we need is some method that can quickly bring two disparate breast shapes into alignment. Here, we take advantage of the popular shape modelling framework of Cootes, et al. [11] to characterize the space of all breast silhouettes.

In the typical shape modelling approach, corresponding landmarks are selected from a large set of training images. Unfortunately, in our case, there are no reliable landmarks on a breast silhouette. Recent techniques in shape modelling, however, allow us to proceed despite this setback. Several groups have begun using non-rigid registration to generate shape models using a pre-labelled 
(1)

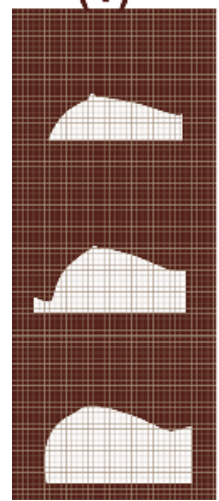

(2)

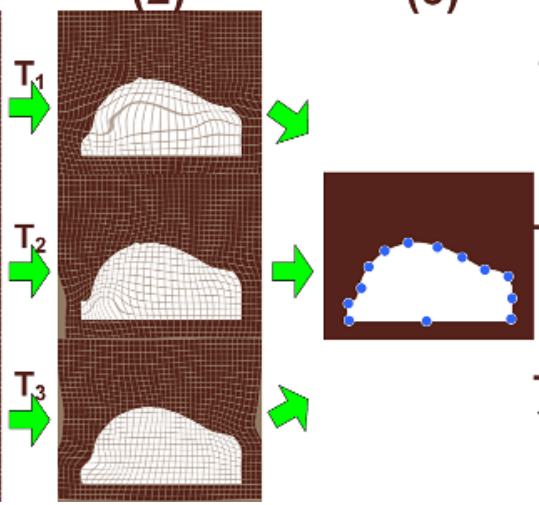

(4)

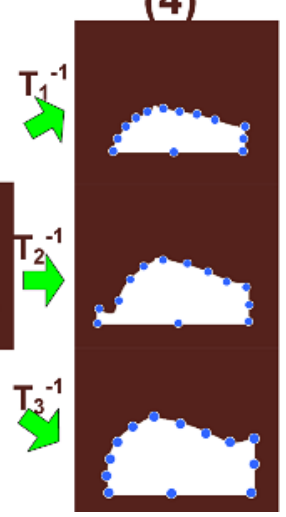

Fig. 1. Basic flow of the use of non-rigid registration for automatic generation of landmark points for shape modelling

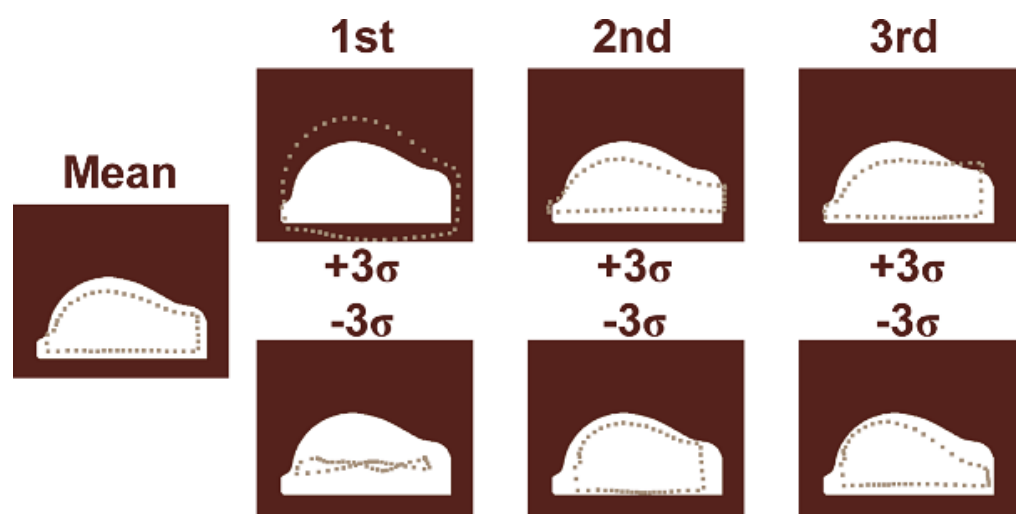

Fig. 2. Mean breast silhouette shape for the MLO view plus the first three principal modes of variation

template [1213]. The basic idea is depicted in Figure 1 which shows how one canonical breast silhouette of the mediolateral oblique (MLO) view can be seeded with pseudo-landmarks which can then be transferred to any other training image via non-rigid registration. The process starts with a typical breast shape upon which some landmarks are placed (column 3). A training set of breast silhouettes (column 1) are then aligned with the canonical shape using nonrigid registration yielding a series of non-rigid transformations (column 2). We can then project the landmarks from the canonical shape back onto the original shape by reversing the corresponding non-rigid transformation (column 4). Given these pseudo-landmark correspondences, we can use the standard principal component analysis paradigm to generate the mean shape and the principal modes of variation as shown in Figure2 2 This figure was generated using a shape parameterization: $\mathbf{S}=\left\{\left(s_{11}, s_{12}\right),\left(s_{11}, s_{12}\right), \ldots,\left(s_{n 1}, s_{n 2}\right)\right\}$ consisting of $n=50$ 
2D points around the breast contour. A training set of 4971 breast silhouettes was used to automatically generate the shape model. Here we see that the first mode corresponds roughly to changes in breast size, the second to length, and the third to general shape. Together, these three modes account for almost $80 \%$ of the variance in the training set. To account for $99 \%$ of the variance in the training set, we only need 17 total modes. We also generated a similar model of the cranio-caudal (CC) view of the breast, trained with 5592 images. Owing to the small range of variability differing $\mathrm{CC}$ views, we only need 6 modes to capture $99.9 \%$ of the training set's variance for the $\mathrm{CC}$ view.

\section{Atlas Generation}

In order to generate a consistent atlas, we must start with a database of mammograms which have been previously marked with instances of cancer. For every mammogram with known cancer, we need to be able to quickly warp it into the atlas shape and record the cancer location in atlas space. To do this, we need to define some transformation function $T$ that can bring different breast shapes into correspondence with the atlas shape. Here, we must not only align the breast silhouettes, we must also be able to match any pixel within the new breast silhouette with a reasonable corresponding pixel in the atlas. To do this we use a two step approach:

1. We use our shape model to bring the new silhouette into alignment with the atlas.

2. We use a very simple triangular mesh together with bilinear interpolation to perform the pixel correspondence.

We will now discuss these two steps in more detail.

For simplicity, for a given view (say, MLO), we choose the atlas shape to be $\mu_{M L O}$, our mean breast shape. Any new breast silhouette can be matched to the mean shape using our shape model which restricts the search space of possible shapes to only those that are probable. Put another way, given $k$ modes of variation $\boldsymbol{\Phi}=\left(\phi_{\mathbf{1}}, \phi_{\mathbf{2}}, \ldots, \phi_{\mathbf{k}}\right)$, we can generate a new MLO breast shape, $\mathbf{S}_{M L O}^{\prime}$ using the equation:

$$
\mathbf{S}_{M L O}^{\prime}=\mathbf{p}+\mu_{M L O}+\sum_{i=1}^{k} \alpha_{i} \phi_{i}
$$

where $\mathbf{p}$ is simply an offset to the $\mu_{M L O}$ to account for a rigid translation of the entire shape. The free parameters in this expression, $\mathbf{p}=\left(p_{x}, p_{y}\right)$ and $\alpha=\alpha_{1}, \alpha_{2}, \ldots, \alpha_{k}$, define the deviations of this shape from the mean along the axes associated with the principal modes. It stands to reason then, that we can perform an optimization over these $k+2$ free parameters to find $\mathbf{S}_{M L O}^{*}$, the optimal matching breast silhouette for any given input.

All we need now is an optimization technique and a cost function. For optimization, we used the simple and popular Nelder-Mead downhill simplex method [14. Our cost function is slightly more complex. Illustrated in Figure $3 a$, it 


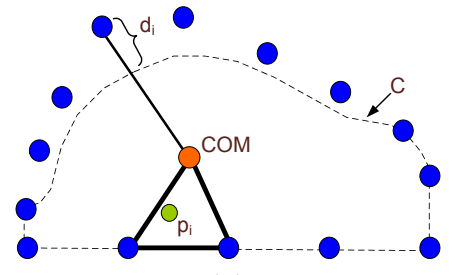

(a)

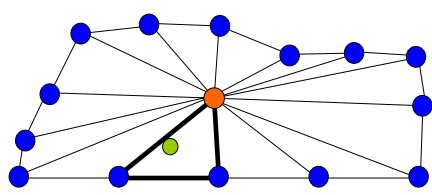

(b)

Fig. 3. (a) Illustration of the cost function for our shape fitting optimization method. (b) Illustration of our point correspondence procedure.

can be described in words as follows. Given a new breast silhouette to fit, we can easily define a chain code $(\mathbf{C})$ around its perimeter. Now, assume we are given a test shape during the optimization, $\mathbf{S}_{M L O}^{t}=\left\{\left(s_{11}^{t}, s_{12}^{t}\right),\left(s_{21}^{t}, s_{22}^{t}\right), \ldots,\left(s_{n 1}^{t}, s_{n 2}^{t}\right)\right\}$, for each point $\mathbf{S}_{M L O}^{t}(i)=\left(s_{i 1}^{t}, s_{i 2}^{t}\right)$, we define $d_{i}$ as the distance between $\mathbf{S}_{M L O}^{t}(i)$ and the intersection between $C$ and the line defined by $\mathbf{S}_{M L O}^{t}(i)$ and $C O M$, the center of mass of the points. The full cost function, therefore, is: $\sum_{i=1}^{n} d_{i}$.

During the optimization, we add one more constraint. As part of the machinery of the PCA, we are given not only the principal modes of variation, $\boldsymbol{\Phi}$, but also their standard deviations, $\boldsymbol{\Sigma}$. We constrain the search for the $\alpha^{\prime} s$ such that $\left|\alpha_{i}\right|<\sqrt{(3)} \sigma_{i}$. This allows us to restrict the search space more stringently to look only at probable breast shapes.

We now need an apparatus to bring individual pixels inside one breast shape into correspondence with another. To do this, we turn each breast into a very simple triangular mesh involving the center of mass and each of the shape points, as shown in Figure 3b. Once we have matched two different breast contours, all pixels within one contour can be mapped into the other, as shown in the following example. The point $p_{i}$ in Figure 3 a can be mapped to its corresponding spot in Figure $3 \mathrm{~b}$ by first identifying the mesh triangle containing $p_{i}$ and then finding its equivalent in the next breast shape. Next, point $p_{i}$ is mapped into its proper place in the new mesh triangle using trilinear interpolation of the vertices of the corresponding triangles.

Now, given a set of mammograms which have had the instances of cancer previously marked by radiologists, we can generate a cancer atlas in the following way. First, we define the atlas shape for a specific view (e.g. MLO or CC) to be the corresponding shape model's mean value, $\mu_{M L O}$. Next, for each mammogram $M_{i}$, we perform these steps:

1. Generate $M_{i}$ 's corresponding silhouette image and associated closed contour.

2. Run the shape fitting optimization to find the $\mathbf{p}^{*}$ and $\alpha^{*}$ which correspond to $\mathbf{S}_{M L O}^{*}$, the optimal fitting shape.

3. Map all pixels corresponding to cancer from $M_{i}$ into the new atlas space.

4. For each cancer pixel mapped into atlas space, increment a counter corresponding to that location.

5. The final, normalized output of the counters corresponds to our probabilistic atlas. 


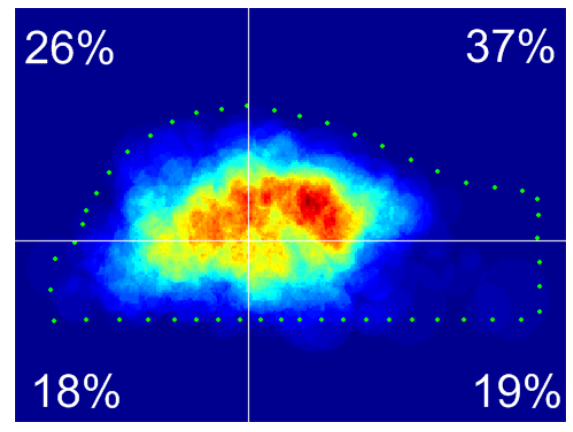

(a)

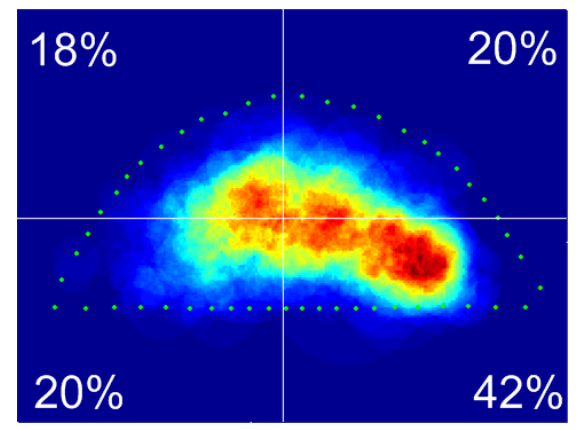

(b)

Fig. 4. (a) Atlas MLO. (b) Atlas CC.

In generating an atlas for both the CC and MLO views, we used 784 digitized and annotated film mammograms from the Digital Database for Screening Mammography (DDSM) 9] as well as 420 digital mammograms from our own database. From the DDSM, we used images from volumes cancer_01, cancer_02, and cancer_05-cancer_15. All cancers used in the atlas generation were selected by radiologists and biopsy proven. In building this atlas, we focused only on cancerous masses, ignoring any mammograms deemed malignant solely because of microcalcifications. Figure 4 shows the results of atlas generation for both the MLO and $\mathrm{CC}$ views. In each of these images, the mean breast shape is superimposed in green and red corresponds to a very high likelihood of cancer, followed by orange, yellow, green, and blue, which indicates a region very unlikely to contain cancer. The first thing to notice about these images is that there do, indeed, seem to be areas of the breast more susceptible to cancer than others. This information will be quite useful when added to a CAD algorithm as we will see in the next section.

\section{Results}

Applying the knowledge encoded in our atlas to a CAD algorithm is relatively straightforward as we can simply add it as a feature to be used in the final classification. More specifically, given a breast lesion candidate detected by Step 1 in the CAD framework discussed in Section 1, we can define the a priori probability that it is cancer using its corresponding view's atlas. Once the breast silhouette containing this new candidate has been aligned with the atlas, the probability can simply be read off from the atlas by projecting the candidate's location into atlas space. This a priori cancer probability is a powerful feature when combined with the other intensity and morphology-based features. It can be used either before, after, or during the classification process.

To test this algorithm, we implemented a CAD scheme very similar to the one in [5], a well-known paper in the CAD field. In this work we are looking only for cancerous masses and not microcalcifications so the candidate detection scheme is based on a combination of pixel intensities and degree of spiculation. Once we 
have the candidates, we segment out the mass regions from the background using a contour-based optimization technique similar to the one in [5]. For the feature generation step of the CAD pipeline, the authors from [5] calculate seven intensitybased features and two density-based features. They then calculate how effective each feature is at classifying candidate masses into benign vs. malignant by combining each feature in turn with a measure of malignancy and a location-based feature, namely the distance of the candidate to the skin line. In this framework, distance to the skin line is the only location-based knowledge used in the classification.

Our hypothesis is that while distance to skin line does add some useful knowledge, when physicians look at a mammogram, they use much more powerful location cues built up over years of experience. We are trying to encode this knowledge as a set of prior probabilities in our cancer atlas. To isolate the effects of location on the classification, we replace the distance to the skin line feature with a feature generated from our atlas. Our feature is the mean a priori probability inside of the mass contour based on our probabilistic atlases. We evaluated our feature using the same basic framework from [5]. We trained a support vector machine (SVM) classifier [15] using each of the nine intensity and density features together with the location-based distance to the skin line feature. We performed the same experiment substituting our probabilistic atlas feature for the location information.

Given this two class problem where class 1 is normal and class 2 is cancer, we set the SVM's $C_{1}$ parameter to be 1 and $C_{2}$ to be $\frac{\text { No. normal training samples }}{\text { No. cancer training samples }}$ We used radial basis functions as the kernel with the $\gamma$ parameter set to the software defaults 15. We trained each SVM with 2430 digitized mammograms consisting of 1204 which contained a biopsy proven cancer (the same database we used to train the cancer atlas itself). We then tested each SVM in turn using a different database, drawn entirely from the DDSM. This time, we used images both from the DDSM cancer volumes as well as the normal volumes. Our testing database consisted of 1732 digitized mammograms, 372 of which contained a biopsy proven cancer. Our testing measure was $A_{z}$, or area under the ROC curve measuring classification performance.

The results are shown below:

\begin{tabular}{|c|c|c|c|c|c|c|c|c|c|}
\hline & con1 & con2 & con3 & con4 & $\operatorname{con} 5$ & $\operatorname{con} 6$ & $\operatorname{con} 7$ & den1 & $\operatorname{den} 2$ \\
\hline feature alone & 78.3 & 53.9 & 78.6 & 79.0 & 75.9 & 55.3 & 82.4 & 78.5 & 76.4 \\
\hline feature + skin line dist. & 79.4 & 57.6 & 79.2 & 78.0 & 76.4 & 59.6 & 82.6 & 78.8 & 76.9 \\
\hline feature + atlas prob. & 82.4 & 66.2 & 80.4 & 80.4 & 78.1 & 67.4 & 84.5 & 80.1 & 77. \\
\hline
\end{tabular}

Here, the seven contrast features from [5] are labelled con1-con7, followed by the two density features. These results suggest that our hypothesis is, indeed, correct. In every case above, the classification score $\left(A_{z}\right)$ improved when using the atlas-based feature, often by a large amount.

In the future, we would like to take this idea further into other CAD fields such as lung cancer and colon cancer. We would also like to explore the improvements we can make by augmenting our shape models with some internal 
anatomical landmarks such as the pectoral muscle. Another area to work on is our very simple internal pixel correspondence model. We would like to look into the improvements we can make by using a more accurate and more complex polygonalization of the inside of the breast. It is also worth noting that this shape correspondence apparatus can be used in a number of other applications such as left-right subtraction of two corresponding breast views and segmentation of the breast region from the background of the mammogram.

\section{References}

1. Cupples, T.E., Cunningham, J.E., Reynolds, J.C.: Impact of computer-aided detection in a regional screening mammography program. Am. J. Roentgenol. 185 (2005) 944-950

2. Karssemeijer, N., Otten, J.D.M., Verbeek, A.L.M., Groenewoud, J.H., de Koning, H.J., Hendriks, J.H.C.L., Holland, R.: Computer-aided detection versus independent double reading of masses on mammograms. Radiology 227 (2003) 192-200

3. Ikeda, D.M., Birdwell, R.L., OShaughnessy, K.F., Sickles, E.A., Brenner, R.J.: Computer-aided detection output on 172 subtle findings on normal mammograms previously obtained in women with breast cancer detected at follow-up screening mammography. Radiology 230 (2004) 811-819

4. Giger, M.L., Huo, Z., Kupinski, M.A., Vyborny, C.J.: Computer-aided diagnosis in mammography. In Fitzpatrick, J.M., Sonka, M., eds.: SPIE Handbook on Medical Imaging - Volume II: Medical Image Processing and Analysis. SPIE (2000) 915-1004

5. te Brake, G.M., Karssemeijer, N., Hendriks, J.H.C.L.: An automatic method to discriminate malignant masses from normal tissue in digital mammograms. Physics in Medicine and Biology 45 (2000) 2843-2857

6. Tabár, L.: Teaching course in diagnostic breast imaging. Mammography Education, Inc., Cave Creek, AZ (2004)

7. Tabár, L., Tot, T., Dean, P.B.: Breast Cancer- The Art and Science of Early Detection with Mammography. Thieme Medical Publishers (2005)

8. Hanley, R.S.: Carcinoma of the breast. Ann R. Coll. Surg. Engl. 57 (1975) 59-66

9. Heath, M., Bowyer, K.W., Kopans, D.: Current status of the digital database for screening mammography. In: Digital Mammography. Kluwer Academic Publishers (1998) 457-460

10. Wirth, A., Stapinski, M.: Automated segmentation of digitized mammograms. Academic Radiology 2 (1995) 1-9

11. Cootes, T.F., Cooper, D., Taylor, C.J., Graham, J.: Active shape models- - their training and application. Computer Vision and Image Understanding 61 (1995) $38-59$

12. Rueckert, D., Frangi, A.F., Schnabel, J.A.: Automatic construction of 3d statistical deformation models of the brain using non-rigid registration. IEEE Transactions on Medical Imaging 22 (2003) 1014-1025

13. Heitz, G., Rohlfing, T., Jr., C.R.M.: Automatic generation of shape models using nonrigid registration with a single segmented template mesh. In: Proc. of the Vision, Modelling and Visualization Conf. (2004) 73-80

14. Press, W.H. In: Numerical Recipes in C. The Art of Scientific Computing. Cambridge University Press (1992) 657-706 http://www.cs.brown.edu/research/ vis/results/bibtex/Press-1992-MOD .bib (bibtex: Press-1992-MOD).

15. Chang, C.C., Lin, C.J.: LIBSVM: a library for support vector machines. (2001) Software available at http://www.csie.ntu.edu.tw/ cjlin/libsvm 